\title{
EEG based emotion recognition using entropy features and Bayesian optimized random forest
}

Himanshu Kumar, Biomedical Engineering Group, Department of Applied Mechanics, Indian Institute of Technology Madras, Chennai, India, him241994@gmail.com

Nagarajan Ganapathy, Peter L. Reichertz Institute for Medical Informatics of TU Braunschweig and Hannover Medical School, Braunschweig, Germany, nagarajan.ganapathy@plri.de

Subha D Puthankattil, Department of Electrical Engineering, National Institute of Technology Calicut, Kozhikode, India subhadp@nitc.ac.in

Ramakrishnan Swaminathan, Biomedical Engineering Group, Department of Applied Mechanics, Indian Institute of Technology Madras, Chennai, India, sramki@iitm.ac.in

\section{Introduction}

Emotions are necessary for human intelligence that is defined by perception, focus, and attention. Electroencephalography (EEG) based emotion recognition is widely preferred in psychology due to its non-invasiveness. Also, frontal regionspecific EEG signals have been associated with emotional processing. However, these signals are multicomponent with large inter and intra-subject variations. Hence, feature selection-based optimized machine learning methods can improve the automated analysis of these signals.

\section{Methods}

In this work, an attempt has been made to classify emotional states using entropy-based features and Bayesian optimized random forest. For this, the EEG signals considered from an online public database and the electrodes from prefrontal and frontal regions (Fp1, Fp2, Fz, F3, and F4) are considered. The signals are decomposed into five frequency bands, namely delta (1-4 Hz), theta (4-8 Hz), alpha (8-13 Hz), beta (14-30 Hz), and gamma (30-45 Hz). Three entropy features, namely Dispersion Entropy (DE), Sample Entropy (SE), and Permutation Entropy (PE), are extracted and are dimensionally reduced using Principal Component Analysis (PCA). Further, the reduced features are applied to the Bayesian optimized random forest for the classification.

\section{Results}

The results show that the proposed method can distinguish various emotional states using frontal region-specific EEG signals. Features, namely DE in the gamma band and SE in the alpha band, exhibit a statistically significant $(\mathrm{p}<0.05)$ difference for classifying arousal and valence emotional states. The selected features from PCA yield an F-measure of $73.24 \%$ for arousal and $46.98 \%$ for valence emotional states. Further, the combination of all features yields a higher Fmeasure of $48.13 \%$ for valence emotional states.

\section{Conclusion}

The proposed method is capable of handling multicomponent variations of frontal region-specific EEG signals. Particularly the combination of selected features could be useful to characterize arousal and valence emotional states. 


\section{Carotid Sound Signal Artifact Detection based on Discrete Wavelet Transform Decomposition}

Patricio Fuentealba, IDTM GmbH, Recklinghausen, Germany + Instituto de Electricidad y Electrónica, Facultad de Ciencias de la Ingeniería, Universidad Austral de Chile, Valdivia, Chile; pfuentealba@uach.cl

Rutuja Salvi, IDTM GmbH, Recklinghausen, Germany, salvi@idtmt.de

Jasmin Henze, Fraunhofer Institute for Software and Systems Engineering, Dortmund, Germany, jasmin.henze@isst.fraunhofer.de

Anja Burmann, Fraunhofer Institute for Software and Systems Engineering, Dortmund, Germany, anja.burmann@isst.fraunhofer.de

Axel Boese, HealthTec Innovation Laboratory, Otto-von-Guericke-University, Magdeburg + MEDICS GmbH, Magdeburg, Germany, axel.boese@med.ovgu.de

Moritz Spiller, HealthTec Innovation Laboratory, Surgical Audio Guidance, Otto-von-Guericke-University, Magdeburg, Germany, moritz.spiller@med.ovgu.de

Alfredo Illanes, HealthTec Innovation Laboratory, Surgical Audio Guidance, Otto-von-Guericke-University, Magdeburg, Germany, alfredo.illanes@med.ovgu.de

Michael Friebe, HealthTec Innovation Laboratory, Otto-von-Guericke-University, Magdeburg + IDTM GmbH, Recklinghausen, Germany, info@friebelab.org

\section{Introduction}

Diagnostic tools based on auscultation allow the non-invasive identification of several cerebrovascular diseases such as stenosis, atherosclerosis, or carotid artery distention. Using a stethoscope, clinicians can listen to the carotid artery flow to assess its status. This operation is difficult, highly dependent on the clinician's experience, and involves a slow learning curve. Recent advances in signal processing and machine learning techniques potentially allow a more objective diagnosis of carotid pathologies. In this context, stable signal segments are essential to ensure a correct evaluation. However, signal artifacts produced by swallowing, coughing, or abrupt movements are inevitable during the signal acquisition, affecting its assessment.

\section{Methods}

The idea is to identify the signal artifacts described above and select informative data for reliable processing. For this purpose, we use real data sampled at $16 \mathrm{kHz}$, acquired with a computer-assisted system consisting of a MEMS digital microphone and a credit card-sized computer. Using this system, we collected 160 recordings of $60 \mathrm{~s}$ length from four users. Each recording involves episodes of normal breathing, apnea, controlled respiration, swallowing, and coughing, in stages of $12 \mathrm{~s}$ length, respectively. For artifact detection, we first decompose the signal by a discrete wavelet transform (DWT). Then, using a sliding window of $1 \mathrm{~s}$ length, we compute the energy ratio between the DWT scales associated with signal information and those associated with artifacts.

\section{Results}

The computed energy ratio significantly differs between swallowing or coughing segments and those involving only normal breathing, apnea, or controlled respiration, which allows a correct artifact segmentation.

\section{Conclusion}

The proposed processing method allows the automatic identification of signal artifacts produced by swallowing and coughing episodes. As a next step, we propose to extract signal features from the stable segments of the carotid sounds and study their performance in predicting specific cardiovascular pathologies such as stenosis, atherosclerosis, or carotid artery distention. 


\section{Vital Signs and Sensors for Post-Exertional Malaise Prevention}

Felix Wichum, Fraunhofer IMS, 47057 Duisburg, Germany, E-Mail: felix.wichum@ims.fraunhofer.de Christian Wiede: Fraunhofer IMS, 47057 Duisburg, Germany, E-Mail: christian.wiede@ims.fraunhofer.de Karsten Seidl: Fraunhofer IMS, 47057 Duisburg, Germany and Department of Electronic Components and Circuits, University of Duisburg-Essen, Duisburg, Germany, E-Mail: karsten.seidl@ims.fraunhofer.de

\section{Introduction}

Myalgic encephalomyelitis (ME) / chronic fatigue syndrome (CFS) is a disease of the nervous system that leads to profound physical weakness. The number of patients is currently increasing worldwide due to the consequences of chronic COVID-19 syndrome. ME/CFS patients suffer from severe fatigue, autonomic, neurological and immunological symptoms. The core symptom Post-Exertional Malaise (PEM) leads to a general exacerbation after even low levels of exertion. Since there is no approved therapy, patient advocacy groups recommend pacing. This involves monitoring trivial vital signs of the patient to avoid PEM. This paper aims to evaluate the suitability of various vital signs and proposes matching sensors.

\section{Methods}

Via a literature review, different vital signs are compared in terms of their usefulness for PEM prevention. Objective criteria such as the number of publications and the PEM triggers to be covered are included in the evaluation. Recommendations for the use of body-worn sensors are derived from the vital signs and the requirements for real-world use. This takes into account patient needs through an objective evaluation of, among other things, wearer comfort, long-term stability and attachment.

\section{Results}

Even simple pedometers can help to monitor the physical trigger of PEM. Strong emotional reactions can be detected by heart rate monitoring. This can be accomplished using electrocardiography and photoplethysmopgraphy sensors. Smartwatches and chest straps in particular are suitable for easy monitoring of vital signs because they enable long-term measurement without interruption and easy sensor attachment.

\section{Conclusion}

The importance of different vital parameter sensors in the monitoring of PEM is elaborated. In the future, more complex analysis of heart rate variability and respiratory rate could be used to prevent PEM. From this, recommendations for the prevention of PEM and potential Long-Covid consequences can be derived. This may help to mitigate the severity of the disease course. 


\section{Optimization and evaluation of motion sequences of an averaged hu- man motion model by using kinematic techniques and new evaluation methods}

Andreas Kitzig, Hochschule Niederrhein University of Applied Science, Faculty of Electrical Engineering and Computer Science, Krefeld, Germany, andreas.kitzig@hs-niederrhein.de Julia Demmer, Hochschule Niederrhein University of Applied Science, Faculty of Electrical Engineering and Computer Science, Krefeld, Germany, julia.demmer@hs-niederrhein.de

Edwin Naroska, Hochschule Niederrhein University of Applied Science, Faculty of Electrical Engineering and Computer Science, Krefeld, Germany, edwin.naroska@hs-niederrhein.de

Gudrun Stockmanns, Hochschule Niederrhein University of Applied Science, Faculty of Electrical Engineering and Computer Science, Krefeld, Germany, gudrun.stockmanns@hs-niederrhein.de

Reinhard Viga, University of Duisburg-Essen, Department of Electronic Components and Circuits, Duisburg, Germany reinhard.viga@uni-due.de

Anton Grabmaier, Fraunhofer IMS and University of Duisburg-Essen, Department of Electronic Components and Circuits Duisburg, Germany, anton.grabmaier@ims.fraunhofer.de

\section{Introduction}

Biosignal processing, pattern recognition, modelling and simulation require a large amount of reference data, both for the development of new algorithms and for evaluation. Depending on the application, the availability of databases is rather low. In the field of biosignal processing with a focus on the functionalization of (nursing) beds, a database with motion sequences of persons in a nursing bed and a method to create average human motion sequences e.g., for person-independent simulation experiments was presented earlier by our research group. Evaluations revealed that some of the averaged motion patterns contain interfering artifacts that arise after averaging due to the variability of the original motion patterns, and thus are not directly applicable. Therefore, we created additional methods for optimizing, evaluating, and merging averaged sequences.

\section{Methods}

An additional procedure was created that realizes the optimization and merging of averaged motion sequences, but also the suppression of unsuitable motion artifacts, with considerations from the field of kinematics. This is done by a processing chain in which, in addition to kinematic considerations, observations of the process-oriented behaviour of human movements, physiological considerations and appropriate interpolation and filtering methods are incorporated.

Before and after the merging and post-processing steps, the motion sequences must be tested and evaluated for use in simulation tasks. For this purpose, four general evaluation criteria are proposed (temporal change of the body vector, the roughness of the motion sequence, the speed of change of a motion sequence and a measure of volume utilization), which enable an assessment of motion sequences in addition to a visual evaluation.

\section{Results \& Conclusion}

The presented method for combination and optimization was subjected to an evaluation against the four presented criteria. It has been shown that combining and optimizing motion sequences does not result in sequences that are fully comparable to real human motion sequences, but the combined sequences are close to real sequences and can be used for simulation tasks. The suitability of the evaluation criteria was evaluated and confirmed using visual comparisons. The new criteria can contribute to a more objective evaluation of motion sequences. The approach will be further improved in the future, for example by analyzing the spectral composition of the individual angular progressions to detect and suppress disturbing artifacts in an even more precise manner. 


\section{Development of a non-invasive continuous blood pressure method for the perioperative application}

Paul Geus (Biofluid Mechanics Lab, Charité - Universitätsmedizin Berlin), Berlin, Germany, paul-friedrich.geus@charite.de

Daniel Laidig (Control Systems Group, Technische Universität Berlin) Berlin, Germany, laidig@control.tu-berlin.de Patrick Borchers Medical Information Technology, RWTH Aachen University, Aachen, Germany, borchers@hia.rwthaachen.de

Thomas Seel (Department Artificial Intelligence in Biomedical Engineering, Friedrich-Alexander-Universität ErlangenNürnberg) Nuremberg, Germany thomas.seel $@$,fau.de

Johanna Stettin (Biofluid Mechanics Lab, Charité - Universitätsmedizin Berlin), Berlin, Germany, johanna.stet$\underline{\text { tin } @ \text { web.de }}$

Ulrich Kertzscher (Biofluid Mechanics Lab, Charité - Universitätsmedizin Berlin), Berlin, Germany, ulrich.kertzscher@charite.de

\section{Introduction}

In most surgeries, the blood pressure is determined non-invasively with the standard measurement frequency being once per five minutes. This can lead to undetected cumulated periods of hypotension, which is known to increase the risk of stroke and mortality. Therefore, a continuous non-invasive blood pressure measurement method at the radial artery is proposed.

\section{Methods}

The setup consists of a balloon, positioned on top of the radial artery, a downstream-positioned ultrasound sensor, a controller, and a pneumatic unit. The blood pressure is determined by a controller that reduces the flow in the radial artery to a constant target value by pneumatically adjusting the pressure of the balloon positioned on top of the radial artery. The balloon pressure can then be correlated to the blood pressure.

To develop this method, a simulator based on the anatomy of the lower arm was constructed. This simulator can create physiological and pathological flow and pressure curves and is used to evaluate the developed method with stress tests. These stress tests include testing the precision of the method in a mean pressure range of $68 \mathrm{mmHg}$ to $105 \mathrm{mmHg}$ and testing three different thicknesses of the tissue model (between $2.1 \mathrm{~mm}$ and $3.5 \mathrm{~mm}$ ) that surrounds the artery model.

\section{Results}

Following a linear regression analysis $\left(\mathrm{R}^{2}\right.$ systole $=0.91$ and $\mathrm{R}^{2}$ diastole $\left.=0.86\right)$, the mean pressure difference between balloon pressure and simulated blood pressure was $3.80 \mathrm{mmHg}( \pm 4.71 \mathrm{mmHg})$ for the systole and $4.89 \mathrm{mmHg}( \pm 6.06 \mathrm{mmHg})$ for the diastole.

\section{Conclusion}

The precision of the proposed method is within the limits proposed by the Association of the Advancement of Medical Instrumentation. However, the measured flow signal and thus the controller performance is susceptible to small movements of the ultrasound sensor. This needs to be resolved before the system can be tested on patients. 


\section{On modifying a matrix pencil variant of Prony's method by singular values decomposition for signal denoising}

Thomas Schanze, Institute for Biomedical Engineering (IBMT), Faculty of Life Science Engineering (LSE), Technische Hochschule Mittelhessen (THM) - University of Applied Sciences, Gießen, Germany, thomas.schanze@1se.thm.de

\section{Introduction}

The goal of signal denoising is to preserve relevant components while reducing noise. Prony's method and its variants have in common that they construct a series of damped complex exponential functions to approximate a uniformly sampled signal. If a signal is contaminated with noise, this will affect the number of functions needed. This raises the question of how to determine those functions that belong to the original signal. We address this question for a variant of Prony's method.

\section{Methods}

Matrix pencil variants of Prony's method usually transform the signal $x_{n}, n=1, \ldots, N$, into an $(N-p) \times(p+1)$ Hankel matrix $\mathbf{X}$, where order $p$ is the number of Prony parameter sets. A set consists of amplitude, damping factor, frequency, and phase. The next steps are to transfer $\mathbf{X}$ into two matrices, $\mathbf{X}_{1}$ consists of the first $p$ and $\mathbf{X}_{2}$ consists of the last $p$ colums of $\mathbf{X}$, to find the generalized eigenvalues of the matrix pencil $\mathbf{X}_{2}-\lambda \mathbf{X}_{1}$, and to compute the Prony parameters. If $x_{n}$ is noise contaminated, $\mathbf{X}$ is also noise contaminated. Instead of filtering $x_{n}$ in time or frequency domain we suggest to compute the singular value decomposition: $\mathbf{U S V}^{\prime}=\mathbf{X}$. If we set an appropriate number of small values of the diagonal matrix $\mathbf{S}$ to zero, i.e. $\mathbf{S} \rightarrow \mathbf{S}_{\mathrm{d}}$, we obtain the denoised matrix $\boldsymbol{X}_{\mathrm{d}}=\mathbf{U} \mathbf{S}_{\mathrm{d}} \mathbf{V}^{\prime}$. Using $\boldsymbol{X}_{\mathrm{d}}$ instead of $\mathbf{X}$ to calculate the Prony parameters results in a denoised Prony series and hence a denoised signal.

\section{Results}

To quantify the denoising performance, the standard deviation of the difference between original signals (simulated/ECG) and the denoised noisy signals was calculated. We have found that high denoising performance requires a careful model order selection and zeroing. However, classical filtering effects do not occur.

\section{Conclusion}

The presented method indicates the signal denoising potential of a matrix pencil variant of Prony's method modified by singular value decomposition. 